\title{
Parental Acceptance for the Use of Silver Diamine Fluoride on Their Special Health Care-Needs Child's Primary and Permanent Teeth
}

\author{
Marwah Almarwan (D) \\ Amjad Almawash ${ }^{2}$ \\ AlHanouf AlBrekan (iD) 3,4 \\ Sarah Albluwi ${ }^{3,4}$ \\ 'Pediatric Dentistry Section, Dentistry \\ Administration, King Fahad Medical City, \\ Riyadh, I I525, Saudi Arabia; \\ ${ }^{2}$ Department of Pediatric Dentistry and \\ Orthodontics, College of Dentistry, King \\ Saud University, Riyadh, II545, Saudi \\ Arabia; ${ }^{3}$ General Dentistry, Private \\ Practice, Riyadh, II564, Saudi Arabia; \\ ${ }^{4}$ Department of Pediatric Dentistry, \\ Prince Sultan Military Medical City, \\ Riyadh, 12233, Saudi Arabia
}

Correspondence: Marwah Almarwan Pediatric Dentistry Section, Dentistry Administration, King Fahad Medical City, Riyadh, II525, Saudi Arabia Tel +966505251667

Email Marwah.almarwan@gmail.com
Purpose: The effectiveness and simplicity of the procedure and the low expense are the main advantages of silver diamine fluoride (SDF). It is useful for controlling caries in challenging patients such as those with special health care needs (SHCN). First aim of this study was to assess parents' acceptance towards using SDF in SHCN children in their primary and permanent teeth. Second aim was to determine the factors that influence parents' decisions toward dental treatment options.

Patients and Methods: This descriptive cross-sectional study included the parents of SHCN children aged 12 years old and younger who were actively seen in pediatric dentistry clinic at King Fahad Medical City (KFMC) and were receiving dental treatment. A previously developed and tested questionnaire was used. The surveyors received training about the questionnaire and participants' consent was obtained before enrolling in the survey. The statistical significance level was set at $\mathrm{P}<0.05$.

Results: Two hundred twelve participants completed the study questionnaire. Parents had a higher acceptance of SDF use regardless of the tooth location and type when their child had a positive history of pain and/or inflammation $(\mathrm{P}=0.019)$. In addition, parents had higher acceptance of SDF staining in primary teeth compared to permanent teeth $(\mathrm{P}=0.006)$. Moreover, the acceptance of SDF staining was higher in posterior teeth compared to anterior teeth in both primary and permanent dentition $(\mathrm{P}<0.001)$.

Conclusion: Parental acceptance of SDF was higher for primary compared to permanent teeth and posterior compared to anterior teeth in both dentitions, as well as for those with a positive history of dental pain and/or inflammation.

Keywords: silver diamine fluoride, special health care needs, parental acceptance, primary teeth, permanent teeth

\section{Introduction}

Dental caries among children is a burden in Saudi Arabia, with an approximate prevalence of $80 \%{ }^{1}$ A national study was conducted in 2010 by Al Dosari et al, which included 11 regions of Saudi Arabia and measured the caries prevalence that was correlated with the fluoridation level in the surveyed area. ${ }^{2}$ Caries in permanent teeth ranged from $59-80 \%$, while in primary teeth, the caries prevalence ranged from $74-90 \% .^{2}$ Another study by Farooqi et al in 2015 reported that the overall prevalence of dental caries lesions in primary and permanent teeth was $73 \%$ in Dammam city, Saudi Arabia. ${ }^{3}$ In Riyadh city, Alhabdan et al in 2018 reported that the prevalence of caries in children aged 6,7 , and 8 years was $87.6 \%, 72.9 \%$, and 
$88.4 \%$, respectively. ${ }^{1}$ Additionally, the prevalence and severity of caries were higher in children from governmental preschools compared to those from private preschools according to Wyne in $2008 .^{4}$

Dental caries is a painful experience for the child, and untreated dental caries consequences could be devastating for the child and their parents/guardians, including but not limited to hospitalizations and emergency room visits, high treatment costs, loss of school days, decreased ability to learn, and reduced oral health-related quality of life. ${ }^{5}$ The treatment and management of dental caries lesions in pre-cooperative children and children with special management considerations (eg, children with special health care needs $[\mathrm{SHCN}]$ ) is challenging. Sometimes advanced pharmacologic behavior guidance modalities (eg, sedation and/or general anesthesia) are required, which may subject children who are less than 3 years old to additional health care risks, as warned by the Food and Drug Administration, with regard to the impairment of brain development in children following exposure to certain anesthetic agents that are used for general anesthesia. ${ }^{6}$ The warning was issued on December 14, 2016, and it recommended that health care providers balance the necessity of appropriate anesthesia in young children especially for procedures that could take more than 3 hours or if multiple procedures are planned in children who are less than 3 years old. ${ }^{6}$

Moreover, accessibility could be difficult, and receiving dental treatment under sedation or general anesthesia might occur in a timely manner (eg, long operating room waiting list or high expenses). ${ }^{7}$

The need for an effective, simple, and inexpensive way to arrest cavitated caries lesions in primary and permanent teeth is of paramount importance, especially for children with SHCN, low-income group children, and patients who have difficulty accessing dental care.

Silver diamine fluoride (SDF) is an inexpensive treatment for dental caries, and it requires low-cost materials and a short chair time for application. ${ }^{7}$ SDF is a topical fluoride solution that is used to arrest caries, and it was approved by the United States Food and Drug Administration as an anti-hypersensitivity agent in $2014 .^{8}$ Laboratory studies found that SDF has an antibacterial effect on cariogenic biofilm, and it stops caries progression. SDF is a safe, reliable, and efficient caries preventive and arresting agent that meets the Millennium Goals of the World Health Organization and the US Institute of Medicine's requirements for twenty-first- century medical care, according to many studies. ${ }^{8}$ The silver in SDF works to inhibit bacterial growth, fluoride induces remineralization, and ammonia stabilizes the high concentrations in solution. ${ }^{8}$

The main drawback of SDF is its non-esthetic result (ie, it permanently stains the enamel and dentinal caries lesions, and it temporarily stains the skin if it is allowed to come into contact, producing a henna-like appearance). However, the American Academy of Pediatric Dentistry conditionally recommends the use of $38 \%$ SDF to manage dental caries and enhance the treatment outcome in children, adolescents, and patients with SHCN. ${ }^{7}$ SDF use was shown to be $89 \%$ more effective for arresting dental caries in the primary teeth than other active treatments. ${ }^{9}$

Parents are an integral part of the process of making decisions towards dental treatment, and thus, their perceptions of the dental treatment are very important. Many studies have been conducted to assess parental acceptance and perception towards SDF, and although the results varied, most of them preferred SDF compared to other invasive treatments such as sedation and general anesthesia, regardless of the tooth-staining effect. ${ }^{10,11}$ In a clinical trial study, $100 \%$ of the parents of SDF-treated pediatric patients did not report any negative impact on their children's esthetic perceptions. ${ }^{12}$ As reported by Clemns et al, SDF application was perceived by most parents as a simple procedure, and they were comfortable with tooth discoloration, the painless process, and the SDF taste. ${ }^{13}$ Most parental acceptance studies towards SDF were performed on healthy pediatric patients, but our first aim in this study is to assess the acceptance of parents towards the use of SDF on their child's primary and permanent teeth in SHCN pediatric patients because they have an increased risk of complications from surgical procedures and need timely treatment. Our second aim is to determine the factors that influence parents' decisions toward dental treatment options.

\section{Materials and Methods}

This descriptive cross-sectional study was conducted at the dentistry administration in King Fahad Medical City (KFMC), Riyadh, Saudi Arabia. The study was approved by the KFMC institutional review board (IRB Log No. 19-486) and followed the Declaration of Helsinki ethical standards for medical research involving human subjects. Informed consent was obtained from all participants before enrolment into the survey. 
The inclusion criteria were as follows: parents of children with SHCN aged 12 years old and younger who attended a pediatric dental clinic at KFMC and agreed to participate in the study. The exclusion criteria were as follows: parents of children with SHCN who were over 12 years old and parents who did not agree to participate in the study. The sample size calculation was performed based on Farooqi et al, ${ }^{3}$ with an overall prevalence of dental caries lesions in primary and permanent teeth of almost $73 \%$ in Dammam city, Saudi Arabia. Using a cumulative caries prevalence of $73 \%$ in the permanent dentition, a confidence interval of $15 \%$ at $95 \%$, and $90 \%$ power, the estimated sample size that was determined using the PASS calculator for single proportion was 146 .

\section{Questionnaire Validation}

A previously developed and tested questionnaire by Bagher et al was used. ${ }^{10}$ The authors agreed to use the questionnaire to reproduce the study results and to compare the results in a different patient group, which was the SHCN population. The surveyors received training to administer the questionnaire.

The first section of the questionnaire included sociodemographic information as well as information about the family's economic status, which was divided into the following three categories: low, moderate, and high. In the second section, parents were asked about their child's cooperation during previous dental visits, the history of pain, and the key determining factor that influenced their decision about the type of dental treatment.

Before COVID-19, the surveyors interviewed the parents at the clinic, and color pictures of primary and permanent teeth that were diagnosed with caries were shown and described before and after treatment with SDF. In addition, the surveyors briefly explained the application steps, costs, advantages, and disadvantages of using SDF. Then, rating the SDF staining acceptability was performed by the participating parents who ranked their acceptance on a five-point Likert scale, as follows: 1, strongly acceptable; 2, acceptable; 3, neutral; 4, unacceptable; and 5, strongly unacceptable They were then asked to consider using SDF for their child's anterior and posterior primary and permanent teeth. The factors upon which the treatment decisions were based were recorded, and information was collected regarding the variables.

During COVID-19, the dental clinic was closed in the middle of March 2020, so patient selection was performed retrospectively from last day of the functional clinic until we completed the data. Fifty-nine patients were surveyed using a printed questionnaire before COVID-19.

To prevent duplication, we reviewed the medical record numbers in the system and compared them to the previously recorded numbers using an Excel data sheet, we confirmed the information and called the patients after verbal consent was obtained. The surveyor then started telephone interviews following all the steps, as previously explained.

\section{Statistical Analysis}

The collected data were statistically analyzed using descriptive statistics. IBM Windows SPSS Software (version 27.0.1.0) was used for the analysis. The Chi-square test was used for nominal data. The statistical significance level was set at $\mathrm{P}<0.05$.

\section{Results}

Two hundred twenty parents provided answers for the questionnaire, and 212 completed it. Most parents were female $149(70 \%)$ and their age ranged from 31 to 40 years $(56 \%)$. For children, the most common age group ranged between 5 and 8 years ( $48 \%$ ). When asked if the child experienced pain or inflammation of the teeth, 153 answered "Yes" (72.2\%). Most of the parents (57\%) answered "No painful procedure" when asked about the most important factor when the child receives a dental treatment. However, about 78 parents (37\%) answered "esthetic" as the important factor. Sociodemographic data are presented in Table 1.

There was a higher rate of parental acceptance of SDF staining regardless of tooth location and type when there was a positive history of tooth pain or inflammation ( $\mathrm{P}=0.019$; Table 2).

Parents' answers to SDF staining acceptability varied according to the tooth type and location, and 60 parents (28.3\%) chose "Acceptable" for anterior primary tooth SDF staining. Moreover, $82(39 \%)$ of the parents answered "Acceptable" for posterior primary tooth SDF staining, whereas $86(41 \%)$ and $67(32 \%)$ of the parents answered "Strongly unacceptable" to anterior and posterior permanent tooth SDF staining, respectively. Generally, parents showed a significantly higher acceptance for SDF staining of primary teeth compared to permanent teeth $(\mathrm{P}=0.006$; Table 3). Furthermore, the acceptance of SDF staining was significantly higher for posterior teeth compared to the anterior teeth in both primary and permanent dentition $(\mathrm{P}<0.001$; Table 3$)$.

Most patients were diagnosed with neurodevelopmental and behavioral disorders (71 patients; 34\%). No 
Table I Sociodemographic Data of Participating Subjects ( $N=2 / 2)$

\begin{tabular}{|l|l|}
\hline Variables & N (\%) \\
\hline Parent Age (Years) & \\
$20-30$ & $17(8 \%)$ \\
$3 I-40$ & $119(56 \%)$ \\
$4 I-60$ & $76(36 \%)$ \\
Mothers & $149(70.3 \%)$ \\
Fathers & $63(30 \%)$ \\
\hline Parental Education & \\
SHigh school & $113(53 \%)$ \\
\hline University and higher education & $99(47 \%)$ \\
\hline Family Income & \\
Low & $37(18 \%)$ \\
Moderate & $139(66 \%)$ \\
High & $36(17 \%)$ \\
\hline Child Age (Years) & \\
I-4 & $20(9.4 \%)$ \\
5-8 & $101(48 \%)$ \\
9-I2 & $91(43 \%)$ \\
Male & $103(49 \%)$ \\
Female & $109(51.4 \%)$ \\
\hline Cooperation during previous dental treatment & \\
Cooperative & $82(39 \%)$ \\
Uncooperative & $130(61 \%)$ \\
\hline History of tooth pain or inflammation & $120(57 \%)$ \\
Yes & $14(7 \%)$ \\
No & $153(72.2 \%)$ \\
I do not know & $51(24 \%)$ \\
\hline Important factor in dental treatment & $8(4 \%)$ \\
Esthetic & \\
\hline
\end{tabular}

significant association was found between medical conditions and the acceptability of SDF staining Table 4.

\section{Discussion}

This research is consistent with Bagher et al's study ${ }^{10}$ in terms of parental acceptance towards the use of SDF on their child's primary and permanent teeth, but the study populations were different. The participants in our study were the parents of SHCN patients who were treated at KFMC, Riyadh, Saudi Arabia. The comparison evaluated SDF use for the primary and permanent teeth and included the tooth location (anterior and posterior).

Significantly, parents were accepting of the SDF staining when there was a positive history of tooth pain and/or inflammation. This finding might be attributed to the probability that parents had experienced either a painful dental procedure or an advanced behavior management technique such as sedation or general anesthesia with their child. Similar to Bagher et al's study, no association was found between the demographic data and the most important treatment goal. ${ }^{10}$ However, most of the parents in our study preferred "non-painful procedure" when asked about the most important goals in dental treatment. This is consistent with other studies in terms of agreeing that SDF causes less discomfort, is more acceptable, and is an alternative to conventional dental treatment. ${ }^{10,14,17}$ Moreover, the absence of pain and ease of the application process were found to be associated with higher parental satisfaction. ${ }^{13} 11$

Additionally, $\mathrm{Hu}$ et al mentioned that treating some children with autism spectrum disorder (ASD) who have a history of dental fear or who have not visited a dentist before can only be accomplished under general anesthesia, thus exposing them to multiple occurrences of dental general anesthesia. ${ }^{17}$ An alternative treatment such as SDF, interim therapeutic restoration, and the Hall technique can effectively and safely manage caries in ASD children. ${ }^{17}$

In our study, parents accepted SDF staining of their child's primary teeth compared to the permanent teeth as well as staining of their posterior teeth compared to the anterior teeth. This is consistent with previous research results, ${ }^{10,15-17}$ and most parents accepted staining on the child's posterior teeth rather than on their anterior teeth. This might be due to parental knowledge that the primary teeth will exfoliate and that staining of the posterior segments are less visible.

There were limitations in this study including the lack of a control group (parents of healthy patients), which limits the

Table 2 Association Between Acceptability of Silver Diamine Fluoride (SDF) Discoloration and History of Pain

\begin{tabular}{|l|l|l|l|l|l|l|}
\hline History of Pain & Strongly Acceptable & Acceptable & Neutral & Unacceptable & Strongly Unacceptable & P-value \\
\hline Yes & $14(9.1 \%)$ & $40(26 \%)$ & $34(22.1 \%)$ & $34(22.1 \%)$ & $32(20.8 \%)$ & 0.019 \\
\hline No & $4(7.3 \%)$ & $19(34.5 \%)$ & $11(20 \%)$ & $16(29.1 \%)$ & $5(9.1 \%)$ & $6(60 \%)$ \\
\hline I do not know & I (10\%) & $3(30 \%)$ & $0(0.0 \%)$ & $0(0.0 \%)$ & \\
\hline
\end{tabular}

Note: Scale scoring was as follows: I, Strongly acceptable; 2, acceptable; 3, neutral; 4, unacceptable; and 5, strongly unacceptable. 
Table 3 Association Between Acceptability of Silver Diamine Fluoride (SDF) Discoloration and Location-Type of Teeth

\begin{tabular}{|c|c|c|c|c|c|c|}
\hline $\begin{array}{l}\text { Location and Type of } \\
\text { Teeth }\end{array}$ & $\begin{array}{l}\text { Strongly } \\
\text { Acceptable }\end{array}$ & Acceptable & Neutral & Unacceptable & $\begin{array}{l}\text { Strongly } \\
\text { Unacceptable }\end{array}$ & P-value \\
\hline Primary anterior teeth & $16(7.5 \%)$ & $60(28.3 \%)$ & 33 (I5.6\%) & $45(21.2 \%)$ & 58 (27.4\%) & \multirow[t]{2}{*}{0.006} \\
\hline Permanent anterior teeth & $12(5.7 \%)$ & 33 (I5.6\%) & 31 (I4.6\%) & $50(23.6 \%)$ & $86(40.6 \%)$ & \\
\hline Primary posterior teeth & $35(16.5 \%)$ & 82 (38.7\%) & 35 (I6.5\%) & 8 (3.8\%) & $52(24.5 \%)$ & \multirow[t]{2}{*}{0.006} \\
\hline Permanent posterior teeth & $23(10.8 \%)$ & $6 \mathrm{I}(28.8 \%)$ & 39 (I8.4\%) & $22(10.4 \%)$ & 67 (31.6\%) & \\
\hline Primary anterior teeth & $16(7.5 \%)$ & $60(28.3 \%)$ & $33(15.6 \%)$ & $45(21.2 \%)$ & $58(27.4 \%)$ & \multirow[t]{2}{*}{0.001} \\
\hline Primary posterior teeth & $35(16.5 \%)$ & $82(38.7 \%)$ & 35 (I6.5\%) & $8(3.8 \%)$ & $52(24.5 \%)$ & \\
\hline Permanent anterior teeth & $12(5.7 \%)$ & $33(15.6 \%)$ & 31 (I4.6\%) & $50(23.6 \%)$ & $86(40.6 \%)$ & \multirow[t]{2}{*}{0.001} \\
\hline Permanent posterior teeth & $23(10.8 \%)$ & $6 \mathrm{I}(28.8 \%)$ & 39 (18.4\%) & $22(10.4 \%)$ & $67(31.6 \%)$ & \\
\hline
\end{tabular}

Note: Scale scoring was as follows: I, Strongly acceptable; 2, acceptable; 3, neutral; 4, unacceptable; and 5, strongly unacceptable.

Table 4 Distribution of Medical Conditions

\begin{tabular}{|l|l|}
\hline Medical Condition & N (\%) \\
\hline Neurodevelopmental and behavioral disorders & $71(34 \%)$ \\
\hline Syndromic disorders & $50(24 \%)$ \\
\hline Craniofacial anomalies & $32(15 \%)$ \\
\hline Pediatric oncology & $15(7 \%)$ \\
\hline Congenital cardiac disease & $10(5 \%)$ \\
\hline Hematological disorders & $10(5 \%)$ \\
\hline Endocrine disorders & $8(4 \%)$ \\
\hline Musculoskeletal disorders & $6(3 \%)$ \\
\hline Renal disorders & $6(3 \%)$ \\
\hline Respiratory diseases & $4(2 \%)$ \\
\hline Total & $212(100 \%)$ \\
\hline
\end{tabular}

generalizability of the results. Also, small sample size from KFMC, which is a governmental hospital where the treatment is provided for free. A multi-center study is required to compare between SHCN and healthy patients. Additionally, the study was interrupted by COVID-19, which delayed and altered the survey. Moreover, none of the parents who participated had a child who received the SDF treatment. Thus, limited access to such treatment may have influenced the parents' decisions and preferences.

As conditionally recommended by the American Academy of Pediatric Dentistry Guidelines, ${ }^{7}$ assessment of the patient's circumstances, preferences, and values by the dentist is important before the intervention. In addition, there should be explanation and consultation with parents or caregivers. ${ }^{7}$ Based on our results, SDF is well accepted by parents of SHCN children because it is simple, arrests caries, does not require advanced behavior guidance modalities, and is cost effective.

\section{Conclusions}

This study showed that there was higher parental acceptance of SDF application when there was a positive history of tooth pain and/or inflammation and that parental acceptance of SDF application was better for primary and posterior teeth compared to permanent and anterior teeth.

\section{Acknowledgment}

We would like to extend our gratitude to Dr. Rana Alsarhan for helping to collect data and interview the participants, and special thanks to the statistician Dr. Turki Almalki.

\section{Funding}

No funding was considered.

\section{Disclosure}

The authors declare that there is no conflict of interest regarding the publication of this paper.

\section{References}

1. Alhabdan YA, Albeshr AG, Yenugadhti N, Jradi H. Prevalence of dental caries and associated factors among primary school children: a population-based cross-sectional study in Riyadh, Saudi Arabia. Environ Health Prev Med. 2018;23(1):60. doi:10.1186/s12199-018$0750-\mathrm{z}$ 
2. Al Dosari AM, Akpata ES, Khan N. Association among dental caries experience, fluorosis and fluoride exposure from drinking water sources in Saudi Arabia. J Public Health Dent. 2010;70:220-226. doi:10.1111/j.1752-7325.2010.00169.x

3. Farooqi FA, Khabeer A, Moheet IA, Khan SQ, Farooq I, AreRjaie AS. Prevalence of dental caries in primary and permanent teeth and its relation with tooth brushing habits among schoolchildren in Eastern Saudi Arabia. Saudi Med J. 2015;36(6):737-742. doi:10.15537/smj.2015.6.10888

4. Wyne AH. Caries prevalence, severity, and pattern in preschool children. J Contemp Dent Pract. 2008;9(3):24-31. doi:10.5005/ jedp-9-3-24

5. American Academy of Pediatric Dentistry. Policy on Early Childhood Caries (ECC): Unique Challenges and Treatment Options. The Reference Manual of Pediatric Dentistry. Chicago, Ill.: American Academy of Pediatric Dentistry; 2020:82-83.

6. Olutoye OA, Baker BW, Belfort MA, Olutoye OO. Food and drug administration warning on anesthesia and brain development: implications for obstetric and fetal surgery. Am J Obstet Gynecol. 2018;218(1):98-102. doi:10.1016/j.ajog.2017.08.107.

7. Crystal YO, Marghalani AA, Ureles SD, et al. Use of silver diamine fluoride for dental caries management in children and adolescents, including those with special health care needs. Pediatr Dent. 2017;39 (5):E135-E145.

8. Horst JA, Ellenikiotis H, Milgrom PM; UCSF Silver Caries Arrest Committee. UCSF protocol for caries arrest using silver diamine fluoride: rationale, indications, and consent. J Calif Dent Assoc. 2016;44(1):16-28

9. Oliveira B, Rajendra A, Veitz-Keenan A, Niederman R. The effect of silver diamine fluoride in preventing caries in the primary dentition: a systematic review and meta-analysis. Caries Res. 2019;53:24-32. doi:10.1159/000488686
10. Bagher SM, Sabbagh HJ, Aljohani SM, Alharbi G, Aldajani M, Elkhodary H. Parental acceptance of the utilization of silver diamine fluoride on their child's primary and permanent teeth. Patient Prefer Adherence. 2019;13:829-835. doi:10.2147/PPA.S205686

11. Magno MB, Silva LP, Ferreira DM, Barja-Fidalgo F, FonsecaGonçalves A. Aesthetic perception, acceptability, and satisfaction in the treatment of caries lesions with silver diamine fluoride: a scoping review. Int J Paediatr Dent. 2019;29:257-266. doi:10.1111/ipd.12465

12. Zhi Q, Lo EM, Lin HC. Randomized clinical trial on effectiveness of silver diamine fluoride and glass ionomer in arresting dentine caries in preschool children. J Dent. 2012;40(11):962-967. doi:10.1016/j. jdent.2012.08.002

13. Clemns J, Gold J, Chaffin J. Effect and acceptance of silver diamine fluoride treatment on dental caries in primary teeth. J Pub Health Dent. 2018;78(1):63-68. doi:10.1111/jphd.12241

14. Mattos-Silveira J, Floriano I, Ferreira FR, Viganó ME, Mendes FM, Braga MM. Children's discomfort may vary among different treatments for initial approximal caries lesions: preliminary findings of a randomized controlled clinical trial. Int J Paediatr Dent. 2015;25 (4):300-304. doi:10.1111/ipd.12134

15. Gordon NB. Silver diamine fluoride staining is acceptable for posterior primary teeth and is preferred over advanced pharmacologic behavior management by many parents. Evid Based Dent Pract. 2018;18:1894-1897.

16. Crystal YO, Janal MN, Hamilton DS, et al. Parental perceptions and acceptance of silver diamine fluoride staining. J Am Dent Assoc. 2017;148:510-518. doi:10.1016/j.adaj.2017.03.013.

17. Hu S, Meyer B, Lai BWP, Chay PL, Tong HJ. Parental acceptance of silver diamine fluoride in children with autism spectrum disorder. Int J Paediatr Dent. 2020;30(4):514-522. doi:10.1111/ipd.12624
Clinical, Cosmetic and Investigational Dentistry

\section{Publish your work in this journal}

Clinical, Cosmetic and Investigational Dentistry is an international, peer-reviewed, open access, online journal focusing on the latest clinical and experimental research in dentistry with specific emphasis on cosmetic interventions. Innovative developments in dental materials, techniques and devices that improve outcomes and patient satisfaction and preference will be highlighted. The manuscript management system is completely online and includes a very quick and fair peer-review system, which is all easy to use. Visit http://www.dovepress.com/testimonials.php to read real quotes from published authors. 